\title{
Calidad de Vida Relacionada con Salud Oral y Autopercepción de Salud: Encuesta Nacional de Calidad de Vida y Salud 2015-2016 en Chile
}

\author{
Quality of Life Related to Oral Health and Self-Perception of Health: \\ National Survey of Quality of Life and Health 2015-2016 in Chile
}

Vezna Sabando ${ }^{1} \&$ Cecilia Albala ${ }^{2}$

SABANDO, V. \& ALBALA, C. Calidad de vida relacionada con salud oral y autopercepción de salud: encuesta nacional de calidad de vida y salud 2015-2016 en Chile. Int. J. Odontostomat., 13(3):338-344, 2019.

RESUMEN: La calidad de vida relacionada con salud oral permite evaluar como los problemas bucodentales pueden ser determinantes el estado de su salud, relaciones sociales, trabajo y bienestar. El objetivo del estudio es determinar la asociación entre la percepción de calidad de vida relacionada a salud oral y la autopercepción de la salud. Estudio transversal de datos de la Encuesta Nacional de Calidad de Vida y Salud (ENCAVI 2015-2016) del Ministerio de Salud, de personas mayores de 15 años de las 15 regiones de Chile. La calidad de vida relacionada con salud oral se midió a través de la versión abreviada del instrumento Oral Health Impact Profile (OHIP-7). La autopercepción de salud se midió con una pregunta única. Se ajustaron modelos de regresión logística para identificar la asociación en términos de Odds Ratio utilizando STATA v15.0. De 7041 encuestas el 50,8 \% son mujeres, la media de edad fue de 42,3 años (d.t. 0,41), el 60,3 \% tiene menos de 12 años de educación, el $36 \%$ declara tener alguna enfermedad crónica. El 18,5\% percibe que sus problemas de salud oral impactan en su calidad de vida. Los factores que se asociaron significativamente con una mala percepción de calidad de vida relacionada con salud oral fueron mala autopercepción de salud general OR=4,84 (IC95 \% 3,$51 ; 6,67$ ), edad (45-54 años) OR=1,98 (IC95 \% 1,26;3,12) y uso frecuente de medicamentos (>3) OR=3,67 (IC95 \% 2,$17 ; 6$, ). La calidad de vida relacionada con salud oral está asociada con la autopercepción en salud, es decir que una mala autopercepción de salud tiene probablemente más riesgo de tener una peor calidad de vida asociada a salud oral, siendo más fuerte a medida que avanza la edad y con el uso frecuente de medicamentos.

PALABRAS CLAVE: calidad de vida, salud oral, autopercepción de salud.

\section{INTRODUCCIÓN}

Los signos clínicos de una enfermedad representan solo una parte del concepto de salud, actualmente a nivel mundial han aumentado las investigaciones que incluyen la evaluación de aspectos subjetivos del bienestar como consecuencia del cambio del modelo biomédico centrado en la enfermedad, por el modelo biopsicosocial (Baiju et al., 2017). Dentro de este marco, el concepto de calidad de vida definido por la OMS como "la percepción del individuo sobre su posición en la vida, en el contexto de la cultura y el sistema de valores en que vive, en relación con sus objetivos, expectativa, estándares y preocupaciones" (The WHOQOL Group, 1995), es un aporte en la evaluación del impacto de la enfermedad y su tratamiento en la percepción del bienestar y en la identificación de problemas de salud percibidos por la población (Gift \& Atchison, 1995).

La calidad de vida relacionada con salud se refiere a aquellos aspectos del bienestar que son relacionados o afectados por la presencia de una enfermedad o por estar bajo algún tratamiento (Karimi \& Brazier, 2016). Un modelo simple que permite comprender los factores de la calidad de vida relacionada con salud es el modelo de Wilson \& Cleary (1995), en este modelo se puede observar como los factores biológicos y fisiológicos interaccionan con las características personales y del ambiente para desen-

\footnotetext{
${ }^{1}$ Programa de Doctorado en Salud Pública Universidad de Chile, Santiago, Chile.

${ }^{2}$ Instituto de Nutrición y Tecnología de Alimentos (INTA) Universidad de Chile, Santiago, Chile.
} 
cadenar una serie de eventos que comprometen las funciones físicas, sociales y psicosociales y que generan una percepción negativa de la salud lo que sumado a factores no médicos tienen un efecto en la calidad de vida (Fig. 1).

Por otro lado, los estudios realizados en calidad de vida relacionada con salud oral (QoLRSO) han permitido comprender el efecto de la salud oral en la percepción de calidad de vida y como los problemas bucodentales pueden ser determinantes el estado de salud, relaciones sociales, trabajo y bienestar (Locker et al., 2000). Sin embargo, frecuentemente la percepción de la salud oral se limita a la estructura anatómica de la boca olvidando la relación que puede tener con el estado de salud. El término calidad de vida relacionado con salud oral hace énfasis en el impacto de las enfermedades de la cavidad oral en la funcionalidad y el bienestar biopsicosocial del individuo (Baiju et al.).

En este contexto, el objetivo de este estudio es determinar la asociación entre la percepción de calidad de vida relacionada a salud oral y la autopercepción de la salud en la población chilena.

\section{MATERIAL Y MÉTODO}

Estudio transversal efectuado a partir de datos de la Encuesta Nacional de Calidad de Vida y Salud (ENCAVI) realizado por el Ministerio de Salud, en una muestra probabilística, estratificada geográficamente y multietápica de personas de 15 y más años chilenas o extranjeras que residen habitualmente en viviendas particulares ocupadas, localizadas en zonas urbanas y rurales de las quince regiones de Chile, entre noviembre 2015 y enero 2016.

La encuesta incluye 10 módulos que abarcan datos sociodemográficos, edad y autoidentificación indígena, información acerca de salud y trabajo, percepción de bienestar y salud, entorno y participación ciudadana, enfermedades y accidentes, hábitos y apoyo social, sexualidad, educación y ocupación de los integrantes del hogar y condiciones de la vivienda.

En esta encuesta, la calidad de vida relacionado a salud oral fue medido a través del instrumento abreviado Oral Health Impact Profile (OHIP-7), este instrumento originalmente de 49 preguntas ha sido

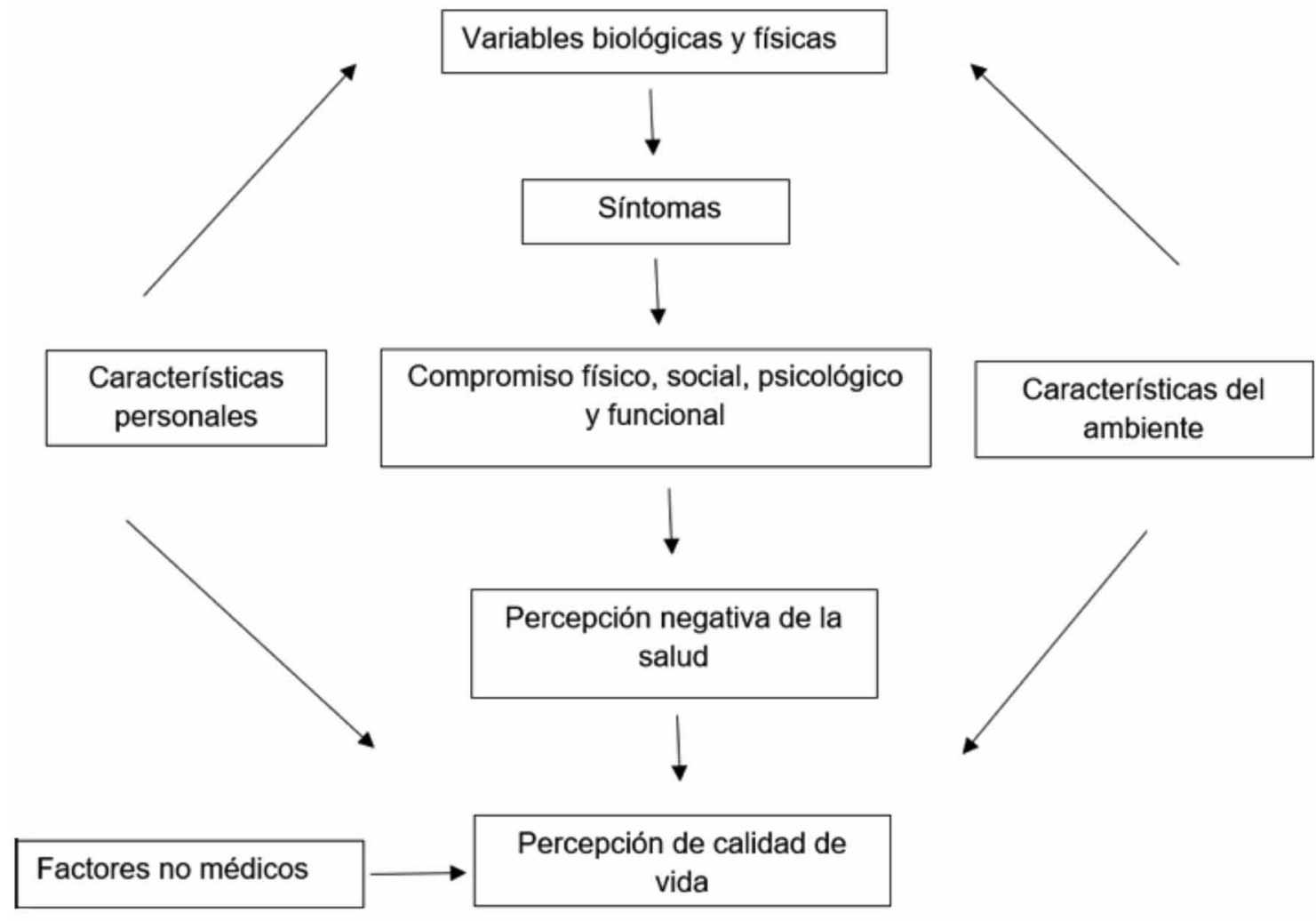

Fig. 1. Modelo de Wilson y Clearly adaptado de Locker et al., 2000. 
adaptado, simplificado y validado en Chile en las versiones abreviadas de 14 y 7 en población adulto mayor (León et al., 2014). El instrumento OHIP-7 contiene 7 ítems relacionados con la frecuencia de impacto adverso causado por la condición de salud oral durante los últimos 12 meses, estos ítems describen las siguientes dimensiones: limitación de funcionalidad, malestar psicológico, discapacidad física, discapacidad psicológica, discapacidad social, dolor físico y minusvalía (Slade \& Spencer, 1994). Cada ítem tiene un puntaje de 1 a 5 en escala Likert donde 1 es "nunca" y 5 "muy frecuente", indicando el grado de severidad en que los individuos perciben su condición de salud oral y síntomas y el efecto en su vida diaria. La suma de los puntajes corresponde a un rango entre 7 y 35 puntos, donde un mayor puntaje es considerado como peor calidad de vida. Se realizó una categorización de los puntajes de cada ítem considerando los puntajes 1 y 2 ("nunca" y "casi nunca") como sin problemas y puntajes de 3 a 5 ("algunas veces", "frecuente" y "muy frecuente") como problemas relacionados con salud oral. Luego al sumar los puntajes de los 7 ítems se obtiene un rango de 0 a 7 , donde el $67,5 \%$ de los individuos obtuvo puntaje 0 , es decir sin problemas relacionados con salud oral. Luego, se tomó como punto de corte el valor 1 , de esta manera se consideró como casos ("Mala calidad de vida relacionada con salud oral") los puntajes mayores e iguales a 2. Esta categorización del OHIP-7 fue utilizado como variables dependientes en los siguientes análisis, este método de análisis fue adaptado de Slade et al. (2005).

La autopercepción de salud se midió a través de una pregunta única la cual tenía 5 tipos de respuestas en escala Likert ("excelente", "muy buena", "buena", "regular", "mala"), las respuestas fueron agrupadas en tres categorías buena, que incluyó las respuestas "excelente", "muy buena" y "buena", y las otras dos categorías "regular" y "mala".

Las edades fueron categorizadas en quinquenios, esto debido a que la percepción de normalidad de una condición física o mental es en relación a un grupo de referencia y está fuertemente influenciado por el ambiente social y cultural (Urzúa, 2010). Las macrozonas son determinadas geográficamente y por densidad de habitantes, dejando a la región Metropolitana que corresponde a la zona centro, como una categoría aparte debido a que concentra gran parte de la población del país.
El nivel educacional se categorizó en menos de 12 años de escolaridad y 12 o más años que corresponde a estudio de enseñanza media completa, técnico o universitario.

El uso de medicamentos de forma cotidiana en diversas patologías se incorporó como una variable importante en el ajuste debido al efecto que tienen los medicamentos consumidos en forma periódica en las condiciones de salud oral especialmente en la disminución del flujo salival, lo que se considera un factor de riesgo para el desarrollo de patologías bucodentales y calidad de vida en salud oral.

Análisis estadístico. El análisis descriptivo de los participantes consideró sus características sociodemográficas: edad, nivel educacional y aspectos generales de calidad de vida, satisfacción con su salud, autoreporte de enfermedades crónicas y autopercepción de salud según sexo, describiendo medidas de frecuencias absolutas y relativas con intervalos de confianza $95 \%$ utilizando método de estimación que consideran el diseño complejo de muestreo, de esta manera primero se establece un modelo "basado en el diseño" según estratos y factores de expansión (comando "svy" para Stata). Para el análisis de QoIRSO por subpoblación se usó test chi^2. Luego, para mejorar la comprensión de la relación entre las variables de percepción de QoIRSO y, autopercepción de salud se realizaron modelos de regresión logística multivariado ajustando por variables sociodemográficas y de salud, identificando la magnitud de asociación en términos de Odds Ratio (OR), la bondad de ajuste del modelo se evaluó a partir de test $\mathrm{F}$-ajustado que considera el diseño de muestra (Archer \& Lemeshow, 2006). Se utilizó el software STATA 15.0.

\section{RESULTADOS}

De un total de 7041 encuestados (que estiman una población de 14.301.195 personas) el 50,8 $\%$ son mujeres. La media de edad fue de 42,3 años (d.t. 0,41). Las edades categorizadas en rangos van entre los $19,2 \%$ para $15-24$ años y $13,4 \%$ para los mayores de 65 años. Dentro de las características de la población el nivel educacional con menos de 12 años de estudio fue el más frecuente $60,3 \%$, sin embargo, es necesario considerar que hay un grupo de menores de 18 años que no alcanzan todavía 
este número de años de escolaridad. En relación con la distribución por zona, la zona centro (restando la Región Metropolitana) presenta una proporción de 33,1\% y la Metropolitana de 40,7 \% coincidiendo con la distribución porcentual nacional. La presencia de enfermedades crónicas en la población es de un $36 \%$, del total de mujeres $43,5 \%$ reportan enfermedades crónicas, mientras que del total de hombres es $28,3 \%(p<0,001)$. El consumo de más de 3 medicamentos de manera frecuente fue de 13,6 $\%$ en la población, en el caso del total de mujeres fue 16 $\%$ y en el total de hombres $10 \%(p<0,0001)$.

Con respecto a la percepción de calidad de vida general a partir de una pregunta única $27,2 \%$ de la población señala que es "regular" y $3,4 \%$ es "mala" y "muy mala" observando diferencias significativas entre hombres y mujeres $(p<0,0001)$. En relación con la autopercepción de salud un $20,9 \%$ considera su salud "regular" y 4,0 \% "mala" en esta categoría se observa que para el total de mujeres la frecuencia es de $5,2 \%$ y en el caso de los hombres el reporte de "mala" salud es de 2,3\% ( $p<0,0001)$.

\section{Mala autopercepción de salud y mala QoIRSO según edad}

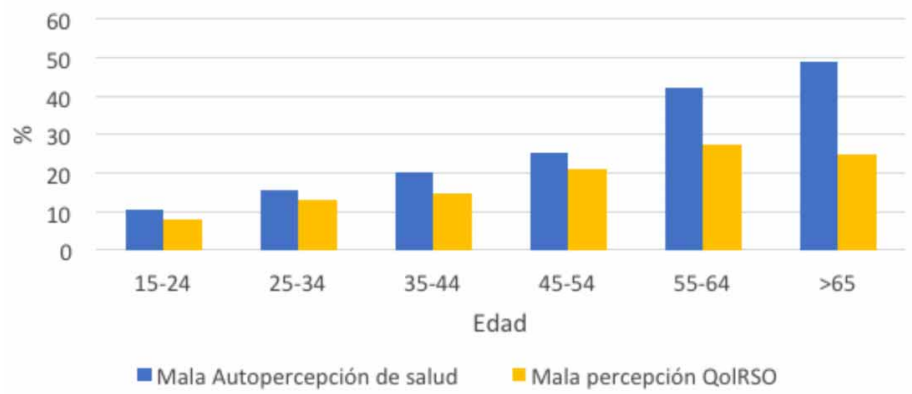

Fig. 2. Frecuencia mala autopercepción de salud y mala QoIRSO según edad.

Buena y regular autopercepción de salud en quienes tienen mala percepción de QolRSO

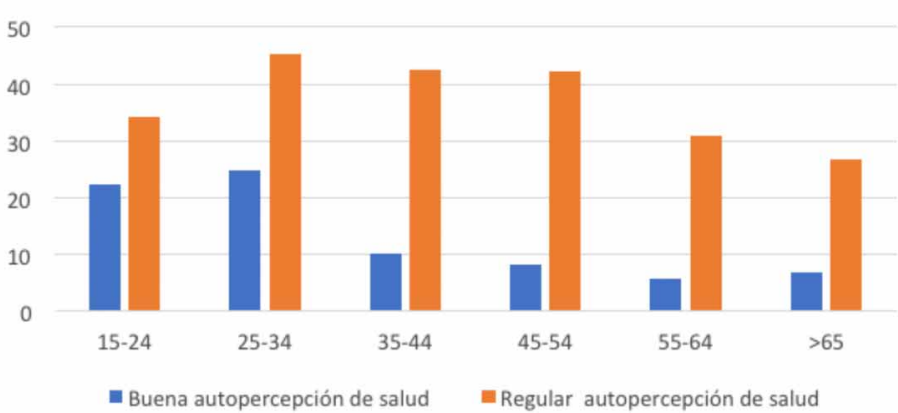

Fig. 3. Frecuencia de Buena y regular autopercepción de salud en quienes tienen mala percepción de QoIRSO.
En el análisis para la QoIRSO se observa que existe diferencia significativa entre los rangos de edades $(p<0,0001)$. Entre los 45 años y 64 años se observa la mayor frecuencia de la mala QoIRSO. En las mujeres la frecuencia de una mala percepción de calidad de vida es casi de $20 \%$ mientras que entre los hombres es de $15 \%(p<0,001)$. En relación con las zonas del país, la zona norte tiene significativamente menor frecuencia de mala percepción de QoIRSO (13,3\%). Entre el área urbana o rural las diferencias de mala percepción de OHQol no son significativa $(p>0,05)$. El uso de más de 3 medicamentos de forma frecuente tiene un porcentaje de $36 \%$ de mala QoLRSO $(p<0,0001)$, la presencia de enfermedades crónicas en personas con mala percepción de QoLRSO fue de un $26 \%(p<0,001)$. Finalmente, se observa que, dentro de las personas con buena autopercepción de la salud, hay un $10 \%$ que evalúan mal su QoLRSO, en aquellas con mala autopercepción de la salud hay $48,2 \%$ que percibe una mala QoLRSO y un tercio de aquellos que perciben tener una regular salud tienen mala evaluación de su salud oral $(p<0,001)$ (Tabla I).

La frecuencia de mala autopercepción de salud y mala percepción de QoIRSO es casi similar en las edades más jóvenes manteniéndose levemente menor la frecuencia de mala percepción de QolRSO. Sin embargo, en el rango de edad entre 45-54 años aumenta la frecuencia de mala percepción de QoLRSO, para luego a partir de los 55 años ser más frecuente la mala autopercepción en salud (Fig. 2).

Para la frecuencia de buena autopercepción de salud en quienes tienen mala percepción de QoIRSO se observa una disminución considerable a partir de los 35 años que continúa hasta los 55-64 años y se mantiene en los mayores de 65 años (Fig. 3).

En el modelo multivariado la mala autopercepción de salud tiene una probabilidad 4,8 veces mayor de presentar una mala calidad de vida asociada a salud oral (IC95 $\% 3,51 ; 6,67)$ lo que se incrementa a medida que avanza la edad y cuando existe consumo frecuente de medicamentos (Tabla II). 
Tabla I. Análisis bivariado para QoIRSO buena y mala según factores de riesgo (tamaño muestral $=6.802$, tamaño estimado de la población=13.906.877).

\begin{tabular}{|c|c|c|c|}
\hline & $\begin{array}{l}\text { Buena } \\
\text { QolRSO \% }\end{array}$ & $\begin{array}{l}\text { Mala } \\
\text { QolRSO \% }\end{array}$ & Valor $p$ \\
\hline & 81,5 & 18,5 & \\
\hline \multicolumn{4}{|l|}{ Edad } \\
\hline $15-24$ & 18,0 & 1,5 & $<0,0001$ \\
\hline $25-34$ & 16,8 & 2,6 & \\
\hline $35-44$ & 15,6 & 2,6 & \\
\hline $45-54$ & 13,6 & 3,6 & \\
\hline $55-64$ & 9,2 & 3,5 & \\
\hline $65+$ & 9,7 & 3,2 & \\
\hline \multicolumn{4}{|l|}{ Sexo } \\
\hline Masculino & 42,0 & 7,4 & $<0,005$ \\
\hline Femenino & 40,9 & 9,6 & \\
\hline \multicolumn{4}{|l|}{ Macrozona } \\
\hline Norte & 10,6 & 1,6 & $<0,005$ \\
\hline Central & 28,3 & 5,1 & \\
\hline Sur & 11,0 & 2,8 & \\
\hline Metropolitana & 32,9 & 7,6 & \\
\hline \multicolumn{4}{|l|}{ Área } \\
\hline Urbana & 71,9 & 14,9 & 0,826 \\
\hline Rural & 10,9 & 2,2 & \\
\hline \multicolumn{4}{|l|}{ Nivel educacional } \\
\hline$\geq 12$ años & 52,4 & 8,4 & $<0,0001$ \\
\hline$<12$ años & 30,6 & 8,7 & \\
\hline \multicolumn{4}{|l|}{$\begin{array}{l}\text { Uso de medicamentos de } \\
\text { forma frecuente }\end{array}$} \\
\hline No & 43,9 & 4,4 & $<0,0001$ \\
\hline $1-2$ & 35,2 & 9,2 & \\
\hline+3 & 4,1 & 3,1 & \\
\hline \multicolumn{4}{|l|}{ Enfermedades Crónicas } \\
\hline No & 56,7 & 7,7 & $<0,0001$ \\
\hline $\mathrm{Si}$ & 26,3 & 9,3 & \\
\hline \multicolumn{4}{|l|}{ Autopercepción de salud } \\
\hline Excelente/Muy buena/buena & 27,1 & 1,9 & $<0,0001$ \\
\hline Regular & 40,3 & 6,3 & \\
\hline Mala & 15,5 & 8,9 & \\
\hline
\end{tabular}

\section{DISCUSIÓN}

Los resultados de este estudio encuentran asociación significativa entre la percepción de calidad de vida relacionada con salud oral y autopercepción de salud, esta última corresponde a la respuesta de cómo considera su estado de salud actual. La importancia de esta asociación está dirigida a la evidencia encontrada entre la relación de autopercepción de salud y mortalidad. El meta-análisis realizado por DeSalvo et al. (2006), señala que una peor autopercepción de salud se relaciona significativamente con el incremento del riesgo de morir.
Una característica particular de la autopercepción de salud en chilenos es la alta frecuencia de respuestas en la categoría regular, especialmente en mujeres, existe evidencia en estudios anteriores del comportamiento particular de esta categoría en especial en personas de mayor edad (Moreno et al., 2017). En el caso de este estudio la categoría regular tiene una magnitud menor de asociación con QoIRSO.

Por otro lado, se observa que la salud oral es parte importante de la salud integral y del bienestar general, las funciones del sistema estomatognático no solo permiten hablar, sonreír, masticar, tragar, sino que además logra transmitir emociones a través de la expresión facial logrando la interacción social fundamental en el desarrollo de la autoestima y la confianza en sí mismos y como consecuencia en percepción de salud y la calidad de vida (Baiju et al.). Sin embargo, la importancia que tiene la salud oral en la autopercepción de salud se ve influenciada por diversos factores, en este estudio esta relación se intensifica a medida que avanza la edad, esto puede deberse al efecto del daño acumulado que presentan especialmente los adultos y adultos mayores en su estado de salud oral y por lo tanto en su calidad de vida (FuentesGarcía et al., 2013), la alta prevalencia de perdida dentaria es un importante factor asociado a la calidad de vida en salud oral y esto a su vez al estado de salud, estado nutricional, perdida de funcionalidad y fragilidad (Netuveli \& Blane, 2008; Kandelman et al., 2008; Hoeksema et al., 2017).

Otro factor que puede influir en una peor condición de salud oral es el uso de medicamentos en forma frecuente, el efecto de su consumo en la disminución del flujo salival aumenta la probabilidad de generar enfermedades bucales y por lo tanto afectar la condición de salud oral y la calidad de vida. El estudio realizado por Gerdin et al. (2005), concluye que tanto la valoración objetiva como subjetiva de la boca seca se asocia con la calidad de vida relacionada con salud oral. 
Tabla II Modelo de regresión logístico multivariado para mala calidad de vida asociada a salud oral y autopercepción de salud.

\begin{tabular}{|c|c|c|c|}
\hline QolRSO (mala) & OR & IC95\% & Valor $p$ \\
\hline \multicolumn{4}{|l|}{ Autopercepción de salud } \\
\hline Excelente/Muy buena/buena & 1 & & \\
\hline Regular & 1,72 & $1,20-2,47$ & $<0,005$ \\
\hline Mala & 4,84 & $3,51-6,67$ & $<0,0001$ \\
\hline \multicolumn{4}{|l|}{ Edad } \\
\hline $15-24$ & 1 & & \\
\hline $25-34$ & 1,50 & $0,93-2,41$ & 0,091 \\
\hline $35-44$ & 1,43 & $0,89-2,31$ & 0,134 \\
\hline $45-54$ & 1,98 & $1,26-3,12$ & 0,003 \\
\hline $55-64$ & 2,15 & $1,22-3,78$ & 0,008 \\
\hline $65+$ & 1,55 & $0,94-2,56$ & 0,08 \\
\hline \multicolumn{4}{|l|}{ Sexo } \\
\hline Hombre & 1 & & \\
\hline Mujer & 0,94 & $0,77-1,17$ & 0,598 \\
\hline \multicolumn{4}{|l|}{ Macrozona } \\
\hline Norte & 1 & & \\
\hline Centro & 0,89 & $0,70-1,14$ & 0,380 \\
\hline Sur & 1,34 & $0,96-1,88$ & 0,084 \\
\hline Metropolitana & 1,32 & $0,99-1,53$ & 0,054 \\
\hline \multicolumn{4}{|l|}{ Nivel educacional } \\
\hline$\geq 12 a$ ños & 1 & & \\
\hline$<12$ años & 1,28 & $0,98-1,66$ & 0,067 \\
\hline \multicolumn{4}{|l|}{ Uso de medicamentos de forma frecuente } \\
\hline Ninguno & 1 & & \\
\hline $1-2$ & 1,82 & $1,44-2,30$ & $<0,0001$ \\
\hline$>3$ & 3,67 & $2,17-6,20$ & $<0,0001$ \\
\hline \multicolumn{4}{|l|}{ Autoreporte de enfermedades crónicas } \\
\hline No & 1 & & \\
\hline $\mathrm{Si}$ & 0,84 & $0,60-1,16$ & 0,289 \\
\hline Test estadístioo F-ajustado (valor $p$ ) & 0,809 & & \\
\hline
\end{tabular}

El número de años de escolaridad también se ha relacionado con el desarrollo de enfermedades bucales. Una mayor educación permite tener más herramientas para el autocuidado de la salud bucal y asegurar la comprensión de la importancia de mantener los dientes hasta edades más avanzadas, permitiendo una mejor nutrición y funcionalidad mejorando la autopercepción de salud y la calidad de vida (Locker et al., 2002). Sin embargo, en este estudio la relación no alcanza la significancia estadística, lo que se puede deber al efecto de la integración de personas jóvenes con menos años de escolaridad, pero con buena percepción de QoIRSO.

Si bien, este estudio no permite determinar la dirección de la asociación, la relevancia de la magnitud sugiere que la preocupación por la condición de salud oral afecta la percepción de la calidad de vida a temprana edad, como se ha visto en estudios realizados en pacientes jóvenes con necesidad de ortodoncia (Hassan \& Amin, 2010) y se hace más evidente en edades avanzadas como consecuencia del efecto acumulado del daño, lo que afecta el estado de salud y la calidad de vida.
SABANDO, V. \& ALBALA, C. Quality of life related to oral health and self-perception of health: national survey of quality of life and health 2015-2016 in Chile. Int. J. Odontostomat., 13(3):338-344, 2019.

ABSTRACT: Quality of life related to oral health assessment oral problems affect an individual's health, social relations, work and well-being. The objective of the study is to determine the association between the perception of the quality of life related with oral health and self-perception of health. A cross-sectional study of data from the National Survey of Quality of Life and Health (2015-2016) of the Ministry of Health was carried out, for people older than 15 years of age, reported from the 15 regions of Chile. Impact profile in oral health (OHIP-7) used to measure Quality of life related to oral health. The self-perception of health was measured with one question. Logistic regression models were adjusted to identify the association in terms of Odds Ratio using STATA v15.0. Out of 7041 surveys, $50.8 \%$ were women, the average age was 42.3 years (d.s., 0.41 ), $60.3 \%$ had less than 12 years of education, $36 \%$ reported having a chronic disease. $18.5 \%$ perceive that their oral health problems impact on their quality of life. Factors that are associated with poor perception of the quality of life related to oral health, were a poor self-perception of health $\mathrm{OR}=4.84$ (95 $\% \mathrm{Cl} 3.51,6.67)$, age $(45-54)$ years) OR = $1.98(95 \% \mathrm{Cl} 1.26,3.12)$ and frequent use of medications (>3) OR $=3.67(95 \% \mathrm{Cl}$ $2.17,6$,). In conclusion, quality of life related to oral health, is directly related to poor selfperception of health and becomes significantly worse with age, as well as with the frequent use of medications.

KEY WORDS: quality of life, oral health, self-perception of health.

\section{REFERENCIAS BILIOGRÁFICAS}

Archer, K. J. \& Lemeshow, S. Goodness-of-fit test for a logistic regression model fitted using survey sample data. Stata J., 6(1):97-105, 2006.

Baiju, R. M.; Peter, E.; Varghese, N. O. \& Sivaram, R. Oral health and quality of life: current concepts. J. Clin. Diagn. Res., 11(6):ZE21-6, 2017. 
DeSalvo, K. B.; Bloser, N.; Reynolds, K.; He, J. \& Muntner, P. Mortality prediction with a single general self-rated health question. A metaanalysis. J. Gen. Intern. Med., 21(3):267-75, 2006.

Fuentes-García, A.; Lera, L.; Sánchez, H. \& Albala, C. Oral healthrelated quality of life of older people from three South American cities. Gerodontology, 30(1):67-75, 2013.

Gerdin, E. W.; Einarson, S.; Jonsson, M.; Aronsson, K. \& Johansson, I. Impact of dry mouth conditions on oral health-related quality of life in older people. Gerodontology, 22(4):219-26, 2005.

Gift, H. C. \& Atchison, K. A. Oral health, health, and health-related quality of life. Med. Care, 33(11 Suppl.):NS57-77, 1995.

Hassan, A. H \& Amin, Hel-S. Association of orthodontic treatment needs and oral health-related quality of life in young adults. Am. J. Orthod. Dentofacial Orthop., 137(1):42-7, 2010.

Hoeksema, A. R.; Spoorenberg, S.; Peters, L. L., Meijer, H., Raghoebar, G. M.; Vissink, A.; Wynia, K. \& Visser, A. Elderly with remaining teeth report less frailty and better quality of life than edentulous elderly: a cross-sectional study. Oral Dis., 23(4):526-36, 2017.

Kandelman, D.; Petersen, P.E, Ueda, H. Oral health, general health, and quality of life in older people. Spec Care Dentist. 28(6):224$36,2008$.

Karimi, M. \& Brazier, J. Health, health-related quality of life, and quality of life: what is the difference? Pharmacoeconomics, 34(7):645-9, 2016.

León, S.; Bravo-Cavicchioli, D.; Correa-Beltrán, G. \& Giacaman, R. A. Validation of the Spanish version of the Oral Health Impact Profile (OHIP-14Sp) in elderly Chileans. B. M. C. Oral Health, 14:95, 2014.

Locker, D.; Clarke, M. \& Payne, B. Self-perceived oral health status, psychological well-being, and life satisfaction in an older adult population. J. Dent. Res., 79(4):970-5, 2000.

Locker, D.; Matear, D.; Stephens, M. \& Jokovic, A. Oral health-related quality of life of a population of medically compromised elderly people. Community Dent. Health, 19(2):90-7, 2002.

Moreno, X.; Albala, C.; Lera, L.; Sánchez, H.; Fuentes-García, A. \& Dangour, A. D. The role of gender in the association between self-rated health and mortality among older adults in Santiago, Chile: A cohort study. PLoS One, 12(7):e0181317, 2017.

Netuveli, G. \& Blane, D. Quality of life in older ages. Br. Med. Bull., 85:113-26, 2008.

Slade, G. D. \& Spencer, A. J. Development and evaluation of the Oral Health Impact Profile. Comunity Dent. Health, 11(1):3-11, 1994.

Slade, G. D.; Nuttall, N., Sanders, A. E.; Steele, J. G.; Allen, P. F. \& Lahti, S. Impacts of oral disorders in the United Kingdom and Australia. Br. Dent. J., 198(8):489-93, 2005.

The WHOQOL Group. The World Health Organization Quality of Life assessment (WHOQOL): position paper from the World Health Organization. Soc. Sci. Med., 41(10):1403-9, 1995.

Urzúa, M. A. Calidad de vida relacionada con la salud: elementos conceptuales. Rev. Med. Chile, 138(3):358-65, 2010.

Wilson, I. B. \& Cleary, P. D. Linking clinical variables with healthrelated quality of life. A conceptual model of patient outcomes. JAMA, 273(1):59-65, 1995.
Dirección para correspondencia:

Vezna Sabando

Programa de Doctorado en Salud Pública

Universidad de Chile

Santiago

CHILE

Email: vmilenka@gmail.com

Received: 02-02-2019

Accepted: 28-03-2019 